\title{
Regular and irregular stimuli result in changes in mice eye movement and cerebellar nuclei neuron model behavior
}

\author{
Tiina Manninen ${ }^{1,2^{*}}$, TD Barbara Nguyen-Vu², Jennifer L Raymond ${ }^{2}$ \\ From 24th Annual Computational Neuroscience Meeting: CNS*2015 \\ Prague, Czech Republic. 18-23 July 2015
}

The cerebellum plays an important role in motor control. The cerebellar key players include Purkinje cells (PCs), mossy fibers, climbing fibers, and parallel fibers. Each $\mathrm{PC}$ receives inputs from many parallel fibers and from a single climbing fiber. Cerebellar outputs originate from the deep cerebellar and vestibular nuclei. Cerebellar nuclei $(\mathrm{CN})$ neurons receive inhibitory inputs from PCs and excitatory inputs from mossy fiber and climbing fiber collaterals. In this work, we studied how regular and irregular, as well as synchronous and asynchronous, PC firing frequencies affect the eye movements in mice and $\mathrm{CN}$ neuron model behavior. Floccular PCs on one side of the head were optogenetically stimulated (see, e.g., [1]). We used both regular and irregular Poisson distributed stimulus trains of $10-90 \mathrm{~Hz}$. As the experimental output, we measured the horizontal eye movements. We compared the effects of the parameters of the stimulus train in the actual eye movements elicited with the effects of the same parameters on the computational $\mathrm{CN}$ neuron model. The $\mathrm{CN}$ neuron model $[2,3]$ includes 517 compartments and it receives an inhibitory input from 450 PC synapses originating from 1-450 individual PCs and an excitatory input from 150 mossy fiber synapses. The model has eight different types of ion channels represented with Hodgkin-Huxley type equations. We used both regular and Poisson distributed PC and gamma distributed mossy fiber spike trains as inputs. We ran the model in NEURON simulation environment [4] and did all the data analysis in MATLAB ${ }^{\circledR}$. We varied the input irregularity, synchrony, mean firing rate $(20-120 \mathrm{~Hz})$, and PC to $\mathrm{CN}$ neuron convergence (1-450). As the model output, we measured the cerebellar nuclei neuron firing rate and mean GABA conductance. The experiments and the model simulations exhibited similar behavior for some but not all of the stimulus frequencies. This suggests a need for further experimental and simulation studies to more fully understand how the parameters of PC firing rate influence downstream oculomotor circuits.

\section{Acknowledgements}

This study was supported by the Academy of Finland (application number 126556), US National Institutes of Health (grant RO1 DC004154), as well as the Emil Aaltonen Foundation and Otto A. Malm Foundation.

\section{Authors' details}

${ }^{1}$ Department of Signal Processing, Tampere University of Technology, Tampere, Finland. ${ }^{2}$ Department of Neurobiology, Stanford School of Medicine, Stanford, CA 94305, USA.

Published: 18 December 2015

\section{References}

1. Nguyen-Vu TB, Kimpo RR, Rinaldi JM, Kohli A, Zeng H, Deisseroth K, Raymond $J$ : Cerebellar Purkinje cell activity drives motor learning. Nat Neurosci 2013, 16(12):1734-1736.

2. Luthman J, Hoebeek FE, Maex R, Davey N, Adams R, De Zeeuw Cl, Steuber V: STD-dependent and independent encoding of input irregularity as spike rate in a computational model of a cerebellar nucleus neuron. Cerebellum 2011, 10(4):667-682.

3. ModelDB, Accession: 144523. [https://senselab.med.yale.edu/ModelDB]

4. Carnevale NT, Hines ML: The NEURON Book. 1st edition. Cambridge: Cambridge University Press; 2006.

doi:10.1186/1471-2202-16-S1-P202

Cite this article as: Manninen et al:: Regular and irregular stimuli result in changes in mice eye movement and cerebellar nuclei neuron model behavior. BMC Neuroscience 2015 16(Suppl 1):P202.

\footnotetext{
* Correspondence: tiina.manninen@tut.fi

'Department of Signal Processing, Tampere University of Technology,

Tampere, Finland

Full list of author information is available at the end of the article
} 